\title{
Adaptive Sliding Mode Control of Uncertain High-Order Nonholonomic Systems with Unknown Control Coefficients
}

\author{
Yan Zhao \\ School of Automation, Southeast University, Nanjing, Jiangsu 210096, China \\ Correspondence should be addressed to Yan Zhao; zyjb03@163.com
}

Received 28 April 2013; Accepted 18 June 2013

Academic Editor: Yuqiang Wu

Copyright ( 2013 Yan Zhao. This is an open access article distributed under the Creative Commons Attribution License, which permits unrestricted use, distribution, and reproduction in any medium, provided the original work is properly cited.

\begin{abstract}
This paper investigates the global stabilization problem for a class of high-order nonholonomic systems with unknown control coefficients and uncertain nonlinearities. An adaptive sliding mode control (SMC) law based on a constructive manipulation is proposed by adding a power integrator technique. A switching control strategy is employed in the control scheme to overcome the uncontrollability problem associated with the nonholonomic systems. The designed sliding mode controller could guarantee the attractiveness of the sliding surface $S=0$ and achieve the asymptotical convergence of the state as well as the boundedness of the estimated parameters. A simulation example is provided to demonstrate the effectiveness of the proposed scheme.
\end{abstract}

\section{Introduction}

Nonholonomic control system as a particular class of nonlinear systems represents a wide class of mechanical systems with nonholonomic (nonintegrable) constraints. The stabilization control for nonholonomic control systems is a formidable problem in the nonlinear control area [1], because the nonholonomic systems cannot be asymptotically stabilized by means of known linear and nonlinear control methods. The difficulty of controlling this class of systems lies in the fact that nonholonomic systems do not satisfy Brockett's necessary conditions for stability as shown in [2]. In the past two decades, many interesting approaches have been proposed to overcome the stabilization obstruction associated with nonholonomic systems, including the smooth timevarying feedback, discontinuous feedback, and nonsmooth time-varying homogeneous feedback; see [1] and references therein.

Variable structure control (VSC) has been shown to be robust in the presence of system variations and external disturbances. During the past few years, the VSC strategy has been also applied to the nonholonomic system control; see [3-9]. It is noted in [5, 6]; a combined adaptive/variable structure control approach was presented for a class of uncertain nonlinear systems. The proposed method not only ensures the convergence to the sliding surface $S=0, \dot{S}=0$, but also retains the asymptotical stability of the classical backstepping procedure. On the basis of the previously proposed control scheme, the so-called second order sliding mode control was exploited for nonholonomic systems in [7]. The work [8] further extended this result to a class of nonholonomic ones with unknown parameters and uncertain nonlinear drifts. In [9], the adaptive SMC problem is studied for a class of perturbed nonholonomic systems which can be transformed into the chained form with uncertain nonlinear drifts.

It should be noted that the results listed previously are all focused on the nonholonomic systems with the affine control variables. High-order nonholonomic systems which include the standard chained form systems as a special case present a new challenge for nonlinear feedback control, since this class of nonholonomic control systems is neither stabilizable by time-invariant continuous state feedback nor affine in the control inputs [10]. By means of adding a power integrator developed in [11], the work [12] first investigated a class of high-order nonholonomic systems in the so-called power chained form. As the further development of this approach, [13] studied a class of uncertain nonholonomic control systems with nonlinear drifts. The recent work [14] removed the assumption of the control gain signs. Reference [15] proposed an adaptive stabilization scheme for a class of high-order 
chained nonholonomic systems without nonlinear drifts in the case of unknown control coefficients.

Motivated by the recent progress in the feedback design for nonholonomic systems, this paper will investigate the global stabilization problem for a class of high order nonholonomic systems with unknown control coefficients and uncertain nonlinearities. A combined backstepping and adaptive SMC methodology developed in $[6,16]$ will be applied to the control design procedure, and adding a power integrator technique is used to deal with the nonaffine control inputs. The tuning function technique presented in [17] is also introduced. We employ a switching control strategy to overcome the stabilization burden associated with the nonholonomic systems.

The paper is organized as follows. Section 2 presents the problem statement. Section 3 gives the SMC design scheme for $x_{0}\left(t_{0}\right) \neq 0$. The switching control strategy and the simulation example are shown in Sections 4 and 5, respectively. Section 6 gives some concluding remarks.

\section{Problem Statement}

In this paper, we consider the following class of high-order nonholonomic systems:

$$
\begin{aligned}
\dot{x}_{0} & =d_{0}(t) u_{0}^{p_{0}}, \\
\dot{x}_{1} & =d_{1}(t) x_{2}^{p_{1}} u_{0}^{q_{1}}+\phi_{1}\left(x_{0}, x_{1}\right), \\
& \vdots \\
\dot{x}_{n-1} & =d_{n-1}(t) x_{n}^{p_{n-1}} u_{0}^{q_{n-1}}+\phi_{n-1}\left(x_{0}, x_{1}, \ldots, x_{n-1}\right), \\
\dot{x}_{n} & =\beta(t) u^{p_{n}}+\phi_{n}\left(x_{0}, x\right),
\end{aligned}
$$

where $\left(x_{0}, x^{T}\right)^{T}=\left(x_{0}, x_{1}, \ldots, x_{n}\right)^{T} \in \mathbb{R}^{n+1}$ are the states, $u_{0}$ and $u$ are two control inputs, $d_{i}(t)(i=0, \ldots, n-1), \beta(t)$ are nonzero functions, and $\phi_{i}: \mathbb{R}^{i+1} \rightarrow \mathbb{R}$ are smooth functions satisfying $\phi_{i}(0, \ldots, 0)=0(i=1, \ldots, n)$. The functions $d_{i}(t)(i=0,1, \ldots, n-1), \beta(t)$ and $\phi_{i}\left(x_{0}, x_{1}, \ldots, x_{i}\right)(i=$ $1, \ldots, n)$ represent the control coefficients and the uncertain nonlinearities, respectively. It is further assumed that $\beta(t)$ is bounded for $t \geq 0, p_{i} \geq 1(i=0, \ldots, n)$ are odd integers, and $q_{k}(k=1, \ldots, n-1)$ are positive integers.

In the rest of this paper, an adaptive sliding mode controller $u$ will be first designed for system (1) such that, given any initial state $\left(x_{0}(0), x(0)\right) \in \Omega_{0}=\left\{\left(x_{0}, x\right) \mid x_{0} \neq 0\right\}$, the closed-loop system is asymptotically stabilized. Then, a switching control strategy is proposed, which guarantees that all the signals are bounded for the initial conditions $\left(x_{0}(0), x(0)\right) \in \mathbb{R}^{n+1}$.

To this end, we make the following assumptions regarding system (1).

Assumption 1. For each $\phi_{i}(i=1, \ldots, n-1)$ in (1), there exist known smooth functions $\varphi_{i}$ satisfying

$$
\phi_{i}\left(x_{0}, x_{1}, \ldots, x_{i}\right) \leq\left(\left|x_{1}\right|+\cdots+\left|x_{i}\right|\right) \varphi_{i}\left(x_{0}, x_{1}, \ldots, x_{i}\right) .
$$

Assumption 2. For each $0 \leq i \leq n-1$, the sign of $d_{i}(t)$ is positive, and there are unknown positive constants $\theta_{1}$ and $\theta_{2}$ such that

$$
\theta_{1} \leq d_{i}(t) \leq \theta_{2}, \quad \forall t \geq 0
$$

Assumption 3. There is a known smooth function $\psi\left(x_{0}, x\right)$ such that $\left|\phi_{n}\left(x_{0}, x\right)\right| \leq \psi\left(x_{0}, x\right)$, for all $\left(x_{0}, x\right) \in \mathbb{R}^{n+1}$.

Next, we introduce a lemma which is crucial in establishing the main results of this paper.

Lemma 4 (Young's Inequality). If the constants $p, q>1$ and satisfy $(p-1)(q-1)=1$, then for all $\varepsilon>0$ and $(x, y) \in \mathbb{R}^{2}$, there holds

$$
x y \leq \frac{\varepsilon^{p}}{p}|x|^{p}+\frac{1}{q \varepsilon^{q}}|y|^{q} .
$$

\section{SMC Applied to Backstepping Design}

In this part, we first assume that $x_{0}\left(t_{0}\right) \neq 0$ for the system (1). The case of $x_{0}\left(t_{0}\right)=0$ will be discussed in the subsequent section.

For $x_{0}\left(t_{0}\right) \neq 0$, take $u_{0}$ as follows:

$$
u_{0}=-x_{0}
$$

Choose the Lyapunov function $V_{0}\left(x_{0}\right)=(1 / 2) x_{0}^{2}$, then the time derivative of $V_{0}\left(x_{0}\right)$ along the first equation of (1) satisfies

$$
\dot{V}_{0}=-d_{0}(t) x_{0}^{p_{0}+1} \leq-\theta_{1} x_{0}^{p_{0}+1} \leq 0
$$

Considering $p_{0}$ is an odd integer, it can be concluded that $x_{0}$ subsystem is asymptotically stable. In addition, $x_{0}\left(t, x_{0}\left(t_{0}\right)\right)$ cannot converge to zero in finite time if $x_{0}\left(t_{0}\right) \neq 0$.

In order to carry out the control design, we introduce the following input-state scaling discontinuous transformation defined by

$$
z_{1}=\frac{x_{1}}{u_{0}^{r_{1}}}, \quad z_{2}=\frac{x_{2}}{u_{0}^{r_{2}}}, \ldots, z_{n}=\frac{x_{n}}{u_{0}^{r_{n}}},
$$

with $r_{i}=q_{i}+p_{i} r_{i+1}, r_{n}=0, \quad i=1, \ldots, n-1$.

Under the control law (5) and the transformation (7), the $x$-subsystem can be transformed into

$$
\begin{aligned}
\dot{z}_{1}= & d_{1}(t) z_{2}^{p_{1}}+d_{0}(t) r_{1} x_{0}^{p_{0}-1} z_{1}+\bar{\phi}_{1}\left(x_{0}, z_{1}\right), \\
\vdots & \\
\dot{z}_{n-1}= & d_{n-1}(t) z_{n}^{p_{n-1}}+d_{0}(t) r_{n-1} x_{0}^{p_{0}-1} z_{n-1} \\
& +\bar{\phi}_{n-1}\left(x_{0}, z_{1}, \ldots, z_{n-1}\right), \\
\dot{z}_{n}= & \beta(t) u^{p_{n}}+\bar{\phi}_{n}\left(x_{0}, z\right),
\end{aligned}
$$

where $\bar{\phi}_{i}\left(x_{0}, z_{1}, \ldots, z_{i}\right)=\phi_{i}\left(x_{0}, x_{1}, \ldots, x_{i}\right) / u_{0}^{r_{i}}, i=1, \ldots, n$. 
To invoke the backstepping method, we define the error variables $\xi_{1}, \ldots, \xi_{n}$ as follows:

$$
\begin{gathered}
\xi_{1}=z_{1}, \\
\xi_{2}=z_{2}^{p_{1}}-\alpha_{1}^{p_{1}}, \ldots, \xi_{n}=z_{n}^{p_{1} \cdots p_{n-1}}-\alpha_{n-1}^{p_{1} \cdots p_{n-1}},
\end{gathered}
$$

where $\alpha_{1}, \ldots, \alpha_{n-1}$ are the virtual controllers and given by

$$
\begin{aligned}
\alpha_{i}^{p_{1} \cdots p_{i}} & \left(x_{0}, z_{1}, \ldots, z_{i}, \widehat{\Theta}\right) \\
& =-\xi_{i} \beta_{i}\left(x_{0}, z_{1}, \ldots, z_{i}, \widehat{\Theta}\right), \quad i=1, \ldots, n-1,
\end{aligned}
$$

where $\beta_{i}\left(x_{0}, z_{1}, \ldots, z_{i}, \widehat{\Theta}\right)>0(i=1, \ldots, n-1)$ are some smooth functions and $\widehat{\Theta}$ is the estimation of unknown parameter $\Theta=\left(1+\theta_{2}^{2 p_{0}}\right) \max \left\{1, \theta_{1}^{-1}\right\}$.

Lemma 5. For every $1 \leq i \leq n-1$, there are smooth nonnegative functions $\bar{\varphi}_{i}\left(x_{0}, z_{1}, \ldots, z_{i}\right)$ satisfying

$\bar{\phi}_{i}\left(x_{0}, z_{1}, \ldots, z_{i}\right) \leq\left(\left|z_{1}\right|+\left|z_{2}\right|+\cdots+\left|z_{i}\right|\right) \bar{\varphi}_{i}\left(x_{0}, z_{1}, \ldots, z_{i}\right)$.

Proof. According to Assumption 1, it can be derived that

$$
\begin{aligned}
\mid \bar{\phi}_{i} & \left(x_{0}, z_{1}, \ldots, z_{i}\right) \mid \\
\leq & u_{0}^{-r_{i}}\left(\left|x_{1}\right|+\cdots+\left|x_{i}\right|\right) \varphi_{i}\left(x_{0}, x_{1}, \ldots, x_{i}\right) \\
= & \left(\left|z_{1}\right|\left|u_{0}\right|^{r_{1}-r_{i}}+\cdots+\left|z_{i-1}\right|\left|u_{0}\right|^{r_{i-1}-r_{i}}+\left|z_{i}\right|\right) \\
& \times \varphi_{i}\left(x_{0}, x_{1}, \ldots, x_{i}\right) \\
\leq & \left(\left|z_{1}\right|+\left|z_{2}\right|+\cdots+\left|z_{i}\right|\right)\left(1+\left.u_{0}\right|^{r_{1}-r_{i}}\right) \\
& \quad \times \varphi_{i}\left(x_{0}, x_{1}, \ldots, x_{i}\right) .
\end{aligned}
$$

In view of $r_{k} \geq r_{i}(k=1, \ldots, i-1)$, one gets (11) with $\bar{\varphi}_{i}=$ $\left(1+\left|u_{0}\right|^{r_{1}-r_{i}}\right) \varphi_{i}$.

In what follows, we give the SMC control design procedure using the recursive method.

Step 1. Choose the candidate Lyapunov function $V_{1}=$ $\left(n / 2 \theta_{1}\right) x_{0}^{2}+(1 / 2) \xi_{1}^{2}+\left(\theta_{1} / 2 \gamma_{1}\right) \widetilde{\Theta}^{2}$ for this step, where $\widetilde{\Theta}=$ $\Theta-\widehat{\Theta}$ is the parameter estimation error, and $\gamma_{1}>0$ is the design gain. Then, along the trajectories of system (8), we have

$$
\begin{aligned}
\dot{V}_{1}= & -\frac{n d_{0}(t)}{\theta_{1}} x_{0}^{p_{0}+1} \\
& +\xi_{1}\left(d_{1}(t) z_{2}^{p_{1}}+d_{0}(t) r_{1} x_{0}^{p_{0}-1} z_{1}+\bar{\phi}_{1}\right)-\frac{\theta_{1}}{\gamma_{1}} \widetilde{\Theta} \dot{\Theta}
\end{aligned}
$$

$$
\begin{aligned}
\leq & -n x_{0}^{p_{0}+1}-\frac{n-1}{\theta_{1}} \xi_{1}^{2}-\xi_{1}^{2}+d_{1}(t) \xi_{1} \xi_{2}+d_{1}(t) \xi_{1} \alpha_{1}^{p_{1}} \\
& +\frac{n-1}{\theta_{1}} \xi_{1}^{2}+\xi_{1}^{2}+\xi_{1} \bar{\phi}_{1} \\
& +d_{0}(t) r_{1} x_{0}^{p_{0}-1} \xi_{1}^{2}-\frac{\theta_{1}}{\gamma_{1}} \widetilde{\Theta} \dot{\Theta} \\
\leq & -n x_{0}^{p_{0}+1}-\frac{n-1}{\theta_{1}} \xi_{1}^{2}-\xi_{1}^{2}+d_{1}(t) \xi_{1} \xi_{2}+d_{1}(t) \xi_{1} \alpha_{1}^{p_{1}} \\
& +\xi_{1}^{2}\left(\frac{n-1}{\theta_{1}}+1+\bar{\varphi}_{1}+d_{0}(t) r_{1} x_{0}^{p_{0}-1}\right)-\frac{\theta_{1}}{\gamma_{1}} \widetilde{\Theta} \dot{\Theta} \\
\leq & -n x_{0}^{p_{0}+1}-\frac{n-1}{\theta_{1}} \xi_{1}^{2}-\xi_{1}^{2}+d_{1}(t) \xi_{1} \xi_{2} \\
& +d_{1}(t) \xi_{1} \alpha_{1}^{p_{1}}+\theta_{1} \Theta \xi_{1}^{2}\left(n+\bar{\varphi}_{1}+r_{1} x_{0}^{p_{0}-1}\right)-\frac{\theta_{1}}{\gamma_{1}} \widetilde{\Theta} \dot{\widehat{\Theta}} .
\end{aligned}
$$

Denote $\rho_{1}\left(x_{0}, z_{1}\right)=n+\bar{\varphi}_{1}+r_{1} x_{0}^{p_{0}-1}, \tau_{1}=\gamma_{1} \xi_{1}^{2} \rho_{1}\left(x_{0}, z_{1}\right)$, and a direct substitution leads to

$$
\begin{aligned}
\dot{V}_{1} \leq & -n x_{0}^{p_{0}+1}-\frac{n-1}{\theta_{1}} \xi_{1}^{2}-\xi_{1}^{2}+d_{1}(t) \xi_{1} \xi_{2}+d_{1}(t) \xi_{1} \alpha_{1}^{p_{1}} \\
& +\theta_{1} \widehat{\Theta} \xi_{1}^{2} \rho_{1}-\frac{\theta_{1}}{\gamma_{1}} \widetilde{\Theta}\left(\dot{\bar{\Theta}}-\tau_{1}\right) .
\end{aligned}
$$

Choose the virtual control law

$$
\begin{aligned}
\alpha_{1}^{p_{1}}\left(x_{0}, z_{1}, \widehat{\Theta}\right) & =-\xi_{1} \rho_{1}\left(x_{0}, z_{1}\right) \sqrt{1+\widehat{\Theta}^{2}} \\
& \triangleq-\xi_{1} \beta_{1}\left(x_{0}, z_{1}, \widehat{\Theta}\right),
\end{aligned}
$$

and one gets

$$
\dot{V}_{1} \leq-n x_{0}^{p_{0}+1}-\frac{n-1}{\theta_{1}} \xi_{1}^{2}-\xi_{1}^{2}+d_{1}(t) \xi_{1} \xi_{2}-\frac{\theta_{1}}{\gamma_{1}} \widetilde{\Theta}\left(\dot{\bar{\Theta}}-\tau_{1}\right) .
$$

Step $i(2 \leq i<n)$. Suppose that at Step $i-1$, there is a positive definite and proper Lyapunov function $V_{i-1}$ satisfying

$$
\begin{aligned}
\dot{V}_{i-1} \leq & -(n-i+2) x_{0}^{p_{0}+1}-\frac{n-i+1}{\theta_{1}} \sum_{k=1}^{i-1} \xi_{k}^{2} \\
& -\sum_{k=1}^{i-1} \xi_{k}^{2}+d_{i-1}(t) \xi_{i-1}^{2-\left(1 / p_{1} \cdots p_{i-2}\right)}\left(z_{i}^{p_{i-1}}-\alpha_{i-1}^{p_{i-1}}\right) \\
& +\left(\tau_{i-1}-\dot{\dot{\Theta}}\right)\left(\frac{\theta_{1}}{\gamma_{1}} \widetilde{\Theta}-\sum_{k=2}^{i-1} \frac{\partial W_{k}}{\partial \widehat{\Theta}}\right) .
\end{aligned}
$$

Let

$$
\begin{gathered}
V_{i}=V_{i-1}+W_{i}, \\
W_{i}=\int_{\alpha_{i-1}}^{z_{i}}\left(s^{p_{1} \cdots p_{i-1}}-\alpha_{i-1}^{p_{1} \cdots p_{i-1}}\right)^{2-\left(1 / p_{1} \cdots p_{i-1}\right)} d s .
\end{gathered}
$$


Then $V_{i}$ is a $C^{2}$, positive definite and proper function such that

$$
\begin{aligned}
\dot{V}_{i}= & \dot{V}_{i-1}+\frac{\partial W_{i}}{\partial x_{0}} \dot{x}_{0}+\sum_{k=1}^{i-1} \frac{\partial W_{i}}{\partial z_{k}} \dot{z}_{k}+\frac{\partial W_{i}}{\partial z_{i}} \dot{z}_{i}+\frac{\partial W_{i}}{\partial \widehat{\Theta}} \\
\leq & -(n-i+2) x_{0}^{p_{0}+1}-\frac{n-i+1}{\theta_{1}} \sum_{k=1}^{i-1} \xi_{k}^{2} \\
& -\sum_{k=1}^{i-1} \xi_{k}^{2}+d_{i-1}(t) \xi_{i-1}^{2-\left(1 / p_{1} \cdots p_{i-2}\right)}\left(z_{i}^{p_{i-1}}-\alpha_{i-1}^{p_{i-1}}\right) \\
& +\frac{\partial W_{i}}{\partial x_{0}} \dot{x}_{0}+\sum_{k=1}^{i-1} \frac{\partial W_{i}}{\partial z_{k}} \dot{z}_{k} \\
& +\xi_{i}^{2-\left(1 / p_{1} \cdots p_{i-1}\right)}\left(\bar{\phi}_{i}+d_{0}(t) r_{i} x_{0}^{p_{0}-1} z_{i}\right) \\
& +d_{i}(t) \xi_{i}^{2-\left(1 / p_{1} \cdots p_{i-1}\right)} z_{i+1}^{p_{i}} \\
& +\left(\tau_{i-1}-\dot{\widehat{\Theta}}\right)\left(\frac{\theta_{1}}{\gamma_{1}} \widetilde{\Theta}-\sum_{k=2}^{i-1} \frac{\partial W_{k}}{\partial \widehat{\Theta}}\right)+\frac{\partial W_{i}}{\partial \widehat{\Theta}} .
\end{aligned}
$$

With the help of Young's Inequality, we can obtain

$$
\begin{aligned}
d_{i-1}( & (t) \xi_{i-1}^{2-\left(1 / p_{1} \cdots p_{i-2}\right)}\left(z_{i}^{p_{i-1}}-\alpha_{i-1}^{p_{i-1}}\right) \\
\leq & 2^{1-\left(1 / p_{1} \cdots p_{i-2}\right)} \theta_{2}\left|\xi_{i-1}\right|^{2-\left(1 / p_{1} \cdots p_{i-2}\right)}\left|\xi_{i}\right|^{1 / p_{1} \cdots p_{i-2}} \\
\leq & \frac{1}{4 \theta_{1}} \xi_{i-1}^{2}+\xi_{i}^{2} \frac{2^{2 p_{1} \cdots p_{i-2}-3}}{p_{1} \cdots p_{i-2}} \\
& \times\left(\frac{4 p_{1} \cdots p_{i-2}-2}{p_{1} \cdots p_{i-2}}\right)^{2 p_{1} \cdots p_{i-2}-1} \theta_{1}^{2 p_{1} \cdots p_{i-2}-1} \theta_{2}^{2 p_{1} \cdots p_{i-2}} \\
\leq & \frac{1}{4 \theta_{1}} \xi_{i-1}^{2}+\theta_{1} \Theta \xi_{i}^{2} \frac{2^{2 p_{1} \cdots p_{i-2}-3}}{p_{1} \cdots p_{i-2}}\left(\frac{4 p_{1} \cdots p_{i-2}-2}{p_{1} \cdots p_{i-2}}\right)^{2 p_{1} \cdots p_{i-2}-1} \\
\triangleq & \frac{1}{4 \theta_{1}} \xi_{i-1}^{2}+\theta_{1} \Theta \xi_{i}^{2} \varrho_{i 1}\left(x_{0}, z_{1}, \ldots, z_{i}, \widehat{\Theta}\right),
\end{aligned}
$$

where $\varrho_{i 1}$ and $\varrho_{i j}(i=2, \ldots, n ; j=1, \ldots, 5)$ given later are smooth nonnegative functions.

From (18), we get

$$
\begin{aligned}
& \frac{\partial W_{i}}{\partial x_{0}} \dot{x}_{0} \\
& =\left(2-\frac{1}{p_{1} \cdots p_{i-1}}\right) d_{0}(t) x_{0}^{p_{0}} \frac{\partial \alpha_{i-1}^{p_{1} \cdots p_{i-1}}}{\partial x_{0}} \\
& \quad \times \int_{\alpha_{i-1}}^{z_{i}}\left(s^{p_{1} \cdots p_{i-1}}-\alpha_{i-1}^{p_{1} \cdots p_{i-1}}\right)^{1-\left(1 / p_{1} \cdots p_{i-1}\right)} d s \\
& \leq\left(4-\frac{2}{p_{1} \cdots p_{i-1}}\right) \theta_{2}\left|x_{0}\right|^{p_{0}}\left|\xi_{i}\right|\left|\frac{\partial \alpha_{i-1}^{p_{1} \cdots p_{i-1}}}{\partial x_{0}}\right|
\end{aligned}
$$

$$
\begin{aligned}
& \leq x_{0}^{p_{0}+1}+\xi_{i}^{2}\left(\left(4-\frac{2}{p_{1} \cdots p_{i-1}}\right) \theta_{2}\left|\frac{\partial \alpha_{i-1}^{p_{1} \cdots p_{i-1}}}{\partial x_{0}}\right|\right)^{p_{0}+1} \xi_{i}^{p_{0}-1} \\
& \leq x_{0}^{p_{0}+1}+\theta_{1} \Theta \xi_{i}^{2}\left(\left(4-\frac{2}{p_{1} \cdots p_{i-1}}\right)\left|\frac{\partial \alpha_{i-1}^{p_{1} \cdots p_{i-1}}}{\partial x_{0}}\right|\right)^{p_{0}+1} \xi_{i}^{p_{0}-1} \\
& \triangleq x_{0}^{p_{0}+1}+\theta_{1} \Theta \xi_{i}^{2} \varrho_{i 2}\left(x_{0}, z_{1}, \ldots, z_{i}, \widehat{\Theta}\right) .
\end{aligned}
$$

In view of

$$
\begin{aligned}
\frac{\partial W_{i}}{\partial z_{k}}= & -\left(2-\frac{1}{p_{1} \cdots p_{i-1}}\right) \frac{\partial \alpha_{i-1}^{p_{1} \cdots p_{i-1}}}{\partial z_{k}} \\
& \times \int_{\alpha_{i-1}}^{z_{i}}\left(s^{p_{1} \cdots p_{i-1}}-\alpha_{i-1}^{p_{1} \cdots p_{i-1}}\right)^{1-\left(1 / p_{1} \cdots p_{i-1}\right)} d s \\
\leq & \left(4-\frac{2}{p_{1} \cdots p_{i-1}}\right)\left|\xi_{i}\right|\left|\frac{\partial \alpha_{i-1}^{p_{1} \cdots p_{i-1}}}{\partial z_{k}}\right|,
\end{aligned}
$$

$$
\begin{aligned}
\frac{\partial \alpha_{i-1}^{p_{1} \cdots p_{i-1}}}{\partial z_{k}} & =-\frac{\partial \xi_{k}}{\partial z_{k}} \beta_{k}-\sum_{l=k}^{i-1} \xi_{l} \frac{\partial \beta_{l}}{\partial z_{k}} \\
& =-p_{1} \cdots p_{k-1} \beta_{k} z_{k}^{p_{1} \cdots p_{k-1}-1}-\sum_{l=k}^{i-1} \xi_{l} \frac{\partial \beta_{l}}{\partial z_{k}},
\end{aligned}
$$

it can be derived that

$$
\begin{aligned}
\sum_{k=1}^{i-1} \frac{\partial W_{i}}{\partial z_{k}} \dot{z}_{k} & \left(4-\frac{2}{p_{1} \cdots p_{i-1}}\right)\left|\xi_{i}\right| \\
& \quad \times \sum_{k=1}^{i-1}\left(p_{1} \cdots p_{k-1} \beta_{k} z_{k}^{p_{1} \cdots p_{k-1}-1}+\sum_{l=k}^{i-1}\left|\xi_{l}\right|\left|\frac{\partial \beta_{l}}{\partial z_{k}}\right|\right)\left|\dot{z}_{k}\right| \\
\leq & \left(4-\frac{2}{p_{1} \cdots p_{i-1}}\right)\left|\xi_{i}\right| \\
& \times \sum_{k=1}^{i-1} p_{1} \cdots p_{k-1} \beta_{k} z_{k}^{p_{1} \cdots p_{k-1}-1} \\
& \times\left(\theta_{2}\left|z_{k+1}\right| p_{k}+\theta_{2} r_{k} x_{0}^{p_{0}-1}\left|z_{k}\right|\right. \\
& +\left(4-\frac{2}{p_{1} \cdots p_{i-1}}\right)\left|\xi_{i}\right|+\cdots+\left|\sum_{k=1}^{i-1} \sum_{l=k}^{i-1}\right| \xi_{l}|| \frac{\partial \beta_{l}}{\partial z_{k}}|| \dot{z}_{k} \mid
\end{aligned}
$$


Mathematical Problems in Engineering

5

$$
\begin{aligned}
& \leq\left(4-\frac{2}{p_{1} \cdots p_{i-1}}\right)\left|\xi_{i}\right| \\
& \leq \frac{1}{4 \theta_{1}} \sum_{k=1}^{i-1} \xi_{k}^{2}+\theta_{1} \Theta \xi_{i}^{2}\left(4-\frac{2}{p_{1} \cdots p_{i-1}}\right)^{2} \\
& \times \sum_{k=1}^{i-1} p_{1} \cdots p_{k-1} \beta_{k} \\
& \times\left(\sum_{k=1}^{i-1} 8\left(p_{1} \cdots p_{k-1} \beta_{k}\right)^{2}\right. \\
& \times\left(\theta_{2} z_{k+1}^{p_{1} \cdots p_{k}}+\theta_{2}\left(1+r_{k} x_{0}^{p_{0}-1}\right) z_{k}^{p_{1} \cdots p_{k-1}}\right. \\
& \cdot\left(\left(2+2 \beta_{k}+2 r_{k} x_{0}^{p_{0}-1}\right)^{2}+\left(\beta_{k}+r_{k+1} x_{0}^{p_{0}-1} \beta_{k}\right)^{2}\right. \\
& +\left(\left|z_{1}\right|^{p_{1} \cdots p_{k-1}}+\cdots+\left|z_{k-1}\right|^{p_{1} \cdots p_{k-1}}\right. \\
& \left.\left.+k\left|z_{k}\right|^{p_{1} \cdots p_{k-1}}\right) \bar{\varphi}_{k}\right) \\
& +2 \sum_{l=k+1}^{i-1}\left(2^{2 p_{k} \cdots p_{l-1}-2} \bar{\varphi}_{k}^{2} \cdot \xi_{k}^{2 p_{k+1} \cdots p_{l-1}-2}\right. \\
& +\left(4-\frac{2}{p_{1} \cdots p_{i-1}}\right)\left|\xi_{i}\right| \sum_{l=1}^{i-1}\left|\xi_{l}\right| \sum_{k=1}^{l}\left|\frac{\partial \beta_{l}}{\partial z_{k}}\right|\left|\dot{z}_{k}\right| \\
& \left.+2^{2 p_{k+1} \cdots p_{l-1}-2} \beta_{k}^{2} \bar{\varphi}_{k+1}^{2} \xi_{k}^{2 p_{k+1} \cdots p_{l-1}-2}\right) \\
& \leq\left(4-\frac{2}{p_{1} \cdots p_{i-1}}\right)\left|\xi_{i}\right| \\
& \left.+2 k^{2}\left(\bar{\varphi}_{k}^{2}+\beta_{k}^{2} \bar{\varphi}_{k+1}^{2}\right)\right) \\
& \left.+\sum_{k=1}^{i-1} \sum_{l=1}^{k}\left(\frac{\partial \beta_{k}}{\partial z_{l}}\right)^{2} \dot{z}_{l}^{2}\right) \\
& \times \sum_{k=1}^{i-1} p_{1} \cdots p_{k-1} \beta_{k} \\
& \times\left(\theta_{2}\left|\xi_{k+1}\right|+\theta_{2}\left(\beta_{k}+1+r_{k} x_{0}^{p_{0}-1}\right)\left|\xi_{k}\right|\right. \\
& \triangleq \frac{1}{4 \theta_{1}} \sum_{k=1}^{i-1} \xi_{k}^{2}+\theta_{1} \Theta \xi_{i}^{2} \varrho_{i 3}\left(x_{0}, z_{1}, \ldots, z_{i}, \widehat{\Theta}\right) \text {. }
\end{aligned}
$$$$
+\theta_{2}\left(1+r_{k} x_{0}^{p_{0}-1}\right) \beta_{k-1}\left|\xi_{k-1}\right|
$$$$
+\sum_{l=1}^{k-1} 2^{p_{l} \cdots p_{k-1}-1}\left(\left|\xi_{l}\right|^{p_{l} \cdots p_{k-1}}+\left|\xi_{l-1} \beta_{l-1}\right|^{p_{l} \cdots p_{k-1}}\right)
$$$$
\left.\times \bar{\varphi}_{l}+k\left(\left|\xi_{k}\right|+\beta_{k-1}\left|\xi_{k-1}\right|\right) \bar{\varphi}_{k}\right)
$$

By Lemma 5, we can obtain

$$
\begin{aligned}
& \xi_{i}^{2-\left(1 / p_{1} \cdots p_{i-1}\right)}\left(\bar{\phi}_{i}+d_{0}(t) r_{i} x_{0}^{p_{0}-1} z_{i}\right) \\
& \leq\left|\xi_{i}\right|^{2-\left(1 / p_{1} \cdots p_{i-1}\right)} \\
& \times\left(\sum_{k=1}^{i}\left|z_{k}\right| \bar{\varphi}_{i}+\theta_{2} r_{i} x_{0}^{p_{0}-1}\right. \\
& \left.\times\left(\left|\xi_{i}\right|^{1 / p_{1} \cdots p_{i-1}}+\left|\xi_{i-1} \beta_{i-1}\right|^{1 / p_{1} \cdots p_{i-1}}\right)\right) \\
& \leq\left|\xi_{i}\right|^{2-\left(1 / p_{1} \cdots p_{i-1}\right)} \\
& \times\left(\sum_{k=1}^{i}\left(\left|\xi_{k}\right|^{1 / p_{1} \cdots p_{k-1}}+\left|\xi_{k-1} \beta_{k-1}\right|^{1 / p_{1} \cdots p_{k-1}}\right)\right. \\
& \times \bar{\varphi}_{i}+\theta_{2} r_{i} x_{0}^{p_{0}-1} \\
& \left.\cdot\left(\left|\xi_{i}\right|^{1 / p_{1} \cdots p_{i-1}}+\left|\xi_{i-1} \beta_{i-1}\right|^{1 / p_{1} \cdots p_{i-1}}\right)\right) \\
& =\left|\xi_{i}\right|^{2-\left(1 / p_{1} \cdots p_{i-1}\right)} \\
& \times \sum_{k=1}^{i-1}\left|\xi_{k}\right|^{1 / p_{1} \cdots p_{i-1}}\left|\xi_{k}\right|^{\left(p_{k} \cdots p_{i-1}-1\right) / p_{1} \cdots p_{i-1}} \\
& \times \bar{\varphi}_{i}+\left|\xi_{i}\right|^{2-\left(1 / p_{1} \cdots p_{i-1}\right)} \\
& \times \sum_{k=1}^{i-1}\left|\xi_{k}\right|^{1 / p_{1} \cdots p_{i-1}} \\
& \cdot\left|\xi_{k}\right|^{\left(p_{k+1} \cdots p_{i-1}-1\right) / p_{1} \cdots p_{i-1}} \beta_{k}^{1 / p_{1} \cdots p_{k}} \bar{\varphi}_{i}
\end{aligned}
$$




$$
\begin{aligned}
& +\theta_{2} r_{i} x_{0}^{p_{0}-1}\left|\xi_{i}\right|^{2-\left(1 / p_{1} \cdots p_{i-1}\right)}\left|\xi_{i-1} \beta_{i-1}\right|^{1 / p_{1} \cdots p_{i-1}} \\
& +\xi_{i}^{2}\left(\bar{\varphi}_{i}+\theta_{2} r_{i} x_{0}^{p_{0}-1}\right) \\
& \leq \frac{1}{4 \theta_{1}} \sum_{k=1}^{i-1} \xi_{k}^{2}+\xi_{i}^{2} \\
& \times\left(\frac{2 p_{1} \cdots p_{i-1}-1}{2 p_{1} \cdots p_{i-1}}\left(\frac{4 \theta_{1}}{p_{1} \cdots p_{i-1}}\right)^{1 /\left(2 p_{1} \cdots p_{i-1}-1\right)}\right. \\
& \times\left(\sum _ { k = 1 } ^ { i - 1 } \left(\left|\xi_{k}\right|^{\left(2 p_{k} \cdots p_{i-1}-1\right) /\left(2 p_{1} \cdots p_{i-1}-1\right)}\right.\right. \\
& +\left|\xi_{k}\right|^{\left(2 p_{k+1} \cdots p_{i-1}-1\right) /\left(2 p_{1} \cdots p_{i-1}-1\right)} \\
& \left.\times \beta_{k}^{2 p_{k+1} \cdots p_{i-1} /\left(2 p_{1} \cdots p_{i-1}-1\right)}\right) \\
& \times \bar{\varphi}_{i}^{2 p_{1} \cdots p_{i-1} /\left(2 p_{1} \cdots p_{i-1}-1\right)} \\
& +\left(\theta_{2} r_{i} x_{0}^{p_{0}-1}\right)^{2 p_{1} \cdots p_{i-1} /\left(2 p_{1} \cdots p_{i-1}-1\right)} \\
& \left.\left.\times \beta_{i-1}^{2 /\left(2 p_{1} \cdots p_{i-1}-1\right)}\right)+\bar{\varphi}_{i}+\theta_{2} r_{i} x_{0}^{p_{0}-1}\right)
\end{aligned}
$$$$
\leq \frac{1}{4 \theta_{1}}
$$$$
\times \sum_{k=1}^{i-1} \xi_{k}^{2}+\theta_{1} \Theta \xi_{i}^{2}
$$$$
\times\left(\left(\frac{4}{p_{1} \cdots p_{i-1}}\right)^{1 /\left(2 p_{1} \cdots p_{i-1}-1\right)}\right.
$$$$
\times\left(\sum _ { k = 1 } ^ { i - 1 } \left(\left|\xi_{k}\right|^{\left(2 p_{k+1} \cdots p_{i-1}-1\right) /\left(2 p_{1} \cdots p_{i-1}-1\right)}\right.\right.
$$$$
\times \beta_{k}^{2 p_{k+1} \cdots p_{i-1} /\left(2 p_{1} \cdots p_{i-1}-1\right)}
$$$$
\left.+\left|\xi_{k}\right|^{\left(2 p_{k} \cdots p_{i-1}-1\right) /\left(2 p_{1} \cdots p_{i-1}-1\right)}\right)
$$$$
\times \bar{\varphi}_{i}^{2 p_{1} \cdots p_{i-1} /\left(2 p_{1} \cdots p_{i-1}-1\right)}
$$$$
+\left(r_{i} x_{0}^{p_{0}-1}\right)^{2 p_{1} \cdots p_{i-1} /\left(2 p_{1} \cdots p_{i-1}-1\right)}
$$$$
\left.\left.\times \beta_{i-1}^{2 /\left(2 p_{1} \cdots p_{i-1}-1\right)}\right)+\bar{\varphi}_{i}+r_{i} x_{0}^{p_{0}-1}\right)
$$$$
\triangleq \frac{1}{4 \theta_{1}} \sum_{k=1}^{i-1} \xi_{k}^{2}+\theta_{1} \Theta \xi_{i}^{2} \varrho_{i 4}\left(x_{0}, z_{1}, \ldots, z_{i}, \widehat{\Theta}\right) .
$$

Substituting (20)-(24) into (19), we have

$$
\dot{V}_{i} \leq-(n-i+1) x_{0}^{p_{0}+1}-\frac{n-i+1}{\theta_{1}} \sum_{k=1}^{i} \xi_{k}^{2}-\sum_{k=1}^{i} \xi_{k}^{2}
$$

$$
\begin{gathered}
+\frac{3}{4 \theta_{1}} \sum_{k=1}^{i-1} \xi_{k}^{2}+\theta_{1} \Theta \xi_{i}^{2}\left(n-i+2+\varrho_{i 1}+\varrho_{i 2}\right. \\
\left.+\varrho_{i 3}+\varrho_{i 4}\right) \\
+d_{i}(t) \xi_{i}^{2-\left(1 / p_{1} \cdots p_{i-1}\right)} z_{i+1}^{p_{i}} \\
+\left(\tau_{i-1}-\dot{\widehat{\Theta}}\right)\left(\frac{\theta_{1}}{\gamma_{1}} \widetilde{\Theta}-\sum_{k=2}^{i-1} \frac{\partial W_{k}}{\partial \widehat{\Theta}}\right)+\frac{\partial W_{i}}{\partial \widehat{\Theta}} \dot{\Theta} .
\end{gathered}
$$

\section{Denote}

$$
\begin{gathered}
\rho_{i}\left(x_{0}, z_{1}, \ldots, z_{i}, \widehat{\Theta}\right)=n-i+2+\varrho_{i 1}+\varrho_{i 2}+\varrho_{i 3}+\varrho_{i 4}, \\
\tau_{i}=\tau_{i-1}+\gamma_{1} \xi_{i}^{2} \rho_{i}\left(x_{0}, z_{1}, \ldots, z_{i}, \widehat{\Theta}\right) .
\end{gathered}
$$

Substituting (26) into (25), we have

$$
\begin{aligned}
\dot{V}_{i} \leq & -(n-i+1) x_{0}^{p_{0}+1}-\frac{n-i+1}{\theta_{1}} \sum_{k=1}^{i} \xi_{k}^{2} \\
& -\sum_{k=1}^{i} \xi_{k}^{2}+\frac{3}{4 \theta_{1}} \sum_{k=1}^{i-1} \xi_{k}^{2} \\
& +d_{i}(t) \xi_{i}^{2-\left(1 / p_{1} \cdots p_{i-1}\right)} z_{i+1}^{p_{i}} \\
& +\theta_{1} \widehat{\Theta} \xi_{i}^{2} \rho_{i}+\left(\tau_{i}-\dot{\widehat{\Theta}}\right)\left(\frac{\theta_{1}}{\gamma_{1}} \widetilde{\Theta}-\sum_{k=2}^{i} \frac{\partial W_{k}}{\partial \widehat{\Theta}}\right) \\
& +\sum_{k=2}^{i-1} \frac{\partial W_{k}}{\partial \widehat{\Theta}}\left(\tau_{i}-\tau_{i-1}\right)+\frac{\partial W_{i}}{\partial \widehat{\Theta}} \tau_{i} .
\end{aligned}
$$

According to (26), there holds

$$
\begin{aligned}
& \sum_{k=2}^{i-1} \frac{\partial W_{k}}{\partial \widehat{\Theta}}\left(\tau_{i}-\tau_{i-1}\right)+\frac{\partial W_{i}}{\partial \widehat{\Theta}} \tau_{i} \\
& \leq \sum_{k=2}^{i-1} \gamma_{1}\left(4-\frac{2}{p_{1} \cdots p_{k-1}}\right)\left|\frac{\partial \alpha_{k-1}^{p_{1} \cdots p_{k-1}}}{\partial \widehat{\Theta}}\right|\left|\xi_{k}\right| \xi_{i}^{2} \rho_{i} \\
& \quad+\gamma_{1}\left(4-\frac{2}{p_{1} \cdots p_{i-1}}\right)\left|\frac{\partial \alpha_{i-1}^{p_{1} \cdots p_{i-1}}}{\partial \widehat{\Theta}}\right|\left|\xi_{i}\right| \sum_{k=1}^{i} \xi_{k}^{2} \rho_{k} \\
& \leq \frac{1}{4 \theta_{1}} \sum_{k=1}^{i-1} \xi_{k}^{2}+\frac{1}{\theta_{1}} \xi_{i}^{2} \\
& \quad+\theta_{1} \xi_{i}^{2}\left(2 \gamma_{1} \sum_{k=2}^{i-1}\left(4-\frac{2}{p_{1} \cdots p_{k-1}}\right)^{2}\left|\frac{\partial \alpha_{k-1}^{p_{1} \cdots p_{k-1}}}{\partial \widehat{\Theta}}\right|^{2} \xi_{i}^{2} \rho_{i}^{2}\right. \\
& \quad+2 \gamma_{1}\left(4-\frac{2}{p_{1} \cdots p_{i-1}}\right)^{2}\left|\frac{\partial \alpha_{i-1}^{p_{1} \cdots p_{i-1}}}{\partial \widehat{\Theta}}\right|^{2} \sum_{k=1}^{i} \xi_{k}^{2} \rho_{k}^{2}
\end{aligned}
$$




$$
\begin{array}{r}
\left.+\frac{\gamma_{1}}{4}\left(4-\frac{2}{p_{1} \cdots p_{i-1}}\right)^{2}\left|\frac{\partial \alpha_{i-1}^{p_{1} \cdots p_{i-1}}}{\partial \widehat{\Theta}}\right|^{2} \xi_{i}^{2} \rho_{i}^{2}\right) \\
\triangleq \frac{1}{4 \theta_{1}} \sum_{k=1}^{i-1} \xi_{k}^{2}+\frac{1}{\theta_{1}} \xi_{i}^{2}+\theta_{1} \xi_{i}^{2} \rho_{i 5}\left(x_{0}, z_{1}, \ldots, z_{i}, \widehat{\Theta}\right) .
\end{array}
$$

As a result, (27) can be further transformed into

$$
\begin{aligned}
\dot{V}_{i} \leq & -(n-i+1) x_{0}^{p_{0}+1}-\frac{n-i}{\theta_{1}} \sum_{k=1}^{i} \xi_{k}^{2} \\
& -\sum_{k=1}^{i} \xi_{k}^{2}+d_{i}(t) \xi_{i}^{2-\left(1 / p_{1} \cdots p_{i-1}\right)} z_{i+1}^{p_{i}} \\
& +\theta_{1} \xi_{i}^{2}\left(\rho_{i} \widehat{\Theta}+Q_{i 5}\right)+\left(\tau_{i}-\dot{\bar{\Theta}}\right)\left(\frac{\theta_{1}}{\gamma_{1}} \widetilde{\Theta}-\sum_{k=2}^{i} \frac{\partial W_{k}}{\partial \widehat{\Theta}}\right) .
\end{aligned}
$$

Choose the $i$ th virtual control law

$$
\begin{aligned}
\alpha_{i}^{p_{1} \cdots p_{i}}\left(x_{0}, z_{1}, \ldots, z_{i}, \widehat{\Theta}\right) & \\
=-\xi_{i}( & \rho_{i}\left(x_{0}, z_{1}, \ldots, z_{i}, \widehat{\Theta}\right) \sqrt{1+\widehat{\Theta}^{2}} \\
& \left.+\varrho_{i 5}\left(x_{0}, z_{1}, \ldots, z_{i}, \widehat{\Theta}\right)\right)^{p_{1} \cdots p_{i-1}},
\end{aligned}
$$

and a direct substitution into (29) results in

$$
\begin{aligned}
\dot{V}_{i} \leq & -(n-i+1) x_{0}^{p_{0}+1}-\frac{n-i}{\theta_{1}} \sum_{k=1}^{i} \xi_{k}^{2} \\
& -\sum_{k=1}^{i} \xi_{k}^{2}+d_{i}(t) \xi_{i}^{2-\left(1 / p_{1} \cdots p_{i-1}\right)}\left(z_{i+1}^{p_{i}}-\alpha_{i}^{p_{i}}\right) \\
& +\left(\tau_{i}-\dot{\widehat{\Theta}}\right)\left(\frac{\theta_{1}}{\gamma_{1}} \widetilde{\Theta}-\sum_{k=2}^{i} \frac{\partial W_{k}}{\partial \widehat{\Theta}}\right)
\end{aligned}
$$

Step $n$. Let

$$
\begin{aligned}
V_{n}= & V_{n-1}+\int_{\alpha_{n-1}}^{z_{n}}\left(s^{p_{1} \cdots p_{n-1}}-\alpha_{n-1}^{p_{1} \cdots p_{n-1}}\right)^{2-\left(1 / p_{1} \cdots p_{n-1}\right)} d s \\
& +\frac{1}{2 \gamma_{2}} \widetilde{\Lambda}^{2},
\end{aligned}
$$

where

$$
\begin{aligned}
\dot{V}_{n-1} \leq & -2 x_{0}^{p_{0}+1}-\frac{1}{\theta_{1}} \sum_{k=1}^{n-1} \xi_{k}^{2} \\
& -\sum_{k=1}^{n-1} \xi_{k}^{2}+d_{n-1}(t) \xi_{n-1}^{2-\left(1 / p_{1} \cdots p_{n-2}\right)}\left(z_{n}^{p_{n-1}}-\alpha_{n-1}^{p_{n-1}}\right) \\
& +\left(\tau_{n-1}-\dot{\widehat{\Theta}}\right)\left(\frac{\theta_{1}}{\gamma_{1}} \widetilde{\Theta}-\sum_{k=2}^{n-1} \frac{\partial W_{k}}{\partial \widehat{\Theta}}\right)
\end{aligned}
$$

$\gamma_{2}>0$ is an arbitrary constant, $\widetilde{\Lambda}=\Lambda-\widehat{\Lambda}$ with $\widehat{\Lambda}$ is the estimate of the unknown constant $\Lambda=\max \left\{\theta_{2}, \theta_{2}^{2 p_{0} p_{1} \cdots p_{n-2}}\right\}$. Choose the parameter update law as

$$
\dot{\widehat{\Theta}}=\tau_{n-1}=\sum_{k=1}^{n-1} \gamma_{1} \xi_{k}^{2} \rho_{k}
$$

and then

$$
\begin{aligned}
\dot{V}_{n}= & \dot{V}_{n-1}+\frac{\partial W_{n}}{\partial x_{0}} \dot{x}_{0}+\sum_{k=1}^{n-1} \frac{\partial W_{n}}{\partial z_{k}} \dot{z}_{k}+\frac{\partial W_{n}}{\partial z_{n}} \dot{z}_{n} \\
& +\frac{\partial W_{n}}{\partial \widehat{\Theta}} \dot{\bar{\Theta}}+\frac{1}{\gamma_{2}} \widetilde{\Lambda} \dot{\bar{\Lambda}} \\
\leq & -2 x_{0}^{p_{0}+1}-\frac{1}{\theta_{1}} \sum_{k=1}^{n-1} \xi_{k}^{2} \\
& -\sum_{k=1}^{n-1} \xi_{k}^{2}+d_{n-1}(t) \xi_{n-1}^{2-\left(1 / p_{1} \cdots p_{n-2}\right)}\left(z_{n}^{p_{n-1}}-\alpha_{n-1}^{p_{n-1}}\right) \\
& +\frac{\partial W_{n}}{\partial x_{0}} \dot{x}_{0}+\sum_{k=1}^{n-1} \frac{\partial W_{n}}{\partial z_{k}} \dot{z}_{k}+\xi_{n}^{2-\left(1 / p_{1} \cdots p_{n-1}\right)} \overline{\phi_{n}} \\
& +d_{n}(t) \xi_{n}^{2-\left(1 / p_{1} \cdots p_{n-1}\right)} u^{p_{n}}+\frac{\partial W_{n}}{\partial \widehat{\Theta}} \dot{\Theta}+\frac{1}{\gamma_{2}} \widetilde{\Lambda} \dot{\bar{\Lambda}} .
\end{aligned}
$$

According to Lemma 4, (9), and (10), we get

$$
\begin{aligned}
d_{n-1}(t) & \xi_{n-1}^{2-\left(1 / p_{1} \cdots p_{n-2}\right)}\left(z_{n}^{p_{n-1}}-\alpha_{n-1}^{p_{n-1}}\right) \\
\leq & \frac{1}{4} \xi_{n-1}^{2}+\xi_{n}^{2} \frac{2^{2 p_{1} \cdots p_{n-2}-3}}{p_{1} \cdots p_{n-2}} \\
& \times\left(\frac{4 p_{1} \cdots p_{n-2}-2}{p_{1} \cdots p_{n-2}}\right)^{2 p_{1} \cdots p_{n-2}-1} \theta_{2}^{2 p_{1} \cdots p_{n-2}} \\
\leq & \frac{1}{4} \xi_{n-1}^{2}+\Lambda \xi_{n}^{2} \frac{2^{2 p_{1} \cdots p_{n-2}-3}}{p_{1} \cdots p_{n-2}}\left(\frac{4 p_{1} \cdots p_{n-2}-2}{p_{1} \cdots p_{n-2}}\right)^{2 p_{1} \cdots p_{n-2}-1} \\
\triangleq & \frac{1}{4} \xi_{n-1}^{2}+\Lambda \xi_{n}^{2} \varrho_{n 1}\left(x_{0}, z, \widehat{\Theta}\right) .
\end{aligned}
$$

By Lemma 4, it can be concluded that

$$
\begin{aligned}
\frac{\partial W_{n}}{\partial x_{0}} \dot{x}_{0} \leq & x_{0}^{p_{0}+1} \\
& +\xi_{n}^{2}\left(\left(4-\frac{2}{p_{1} \cdots p_{n-1}}\right) \theta_{2}\left|\frac{\partial \alpha_{n-1}^{p_{1} \cdots p_{n-1}}}{\partial x_{0}}\right|\right)^{p_{0}+1} \xi_{n}^{p_{0}-1} \\
\leq & x_{0}^{p_{0}+1}+\Lambda \xi_{n}^{2} \\
& \times\left(\left(4-\frac{2}{p_{1} \cdots p_{n-1}}\right)\left|\frac{\partial \alpha_{n-1}^{p_{1} \cdots p_{n-1}}}{\partial x_{0}}\right|\right)^{p_{0}+1} \xi_{n}^{p_{0}-1} \\
\triangleq & x_{0}^{p_{0}+1}+\Lambda \xi_{n}^{2} \varrho_{n 2}\left(x_{0}, z, \widehat{\Theta}\right) .
\end{aligned}
$$


With the same strategy as previously, it follows mentioned that

$$
\begin{aligned}
& \sum_{k=1}^{n-1} \frac{\partial W_{n}}{\partial z_{k}} \dot{z}_{k} \\
& \leq \frac{1}{4} \sum_{k=1}^{n-1} \xi_{k}^{2}+\xi_{n}^{2} 8\left(4-\frac{2}{p_{1} \cdots p_{n-1}}\right)^{2 n-1} \sum_{k=1}^{n}\left(p_{1} \cdots p_{k-1} \beta_{k}\right)^{2} \\
& \cdot\left(\theta_{2}^{2}\left(\left(2+2 \beta_{k}+2 r_{k} x_{0}^{p_{0}-1}\right)^{2}+\left(\beta_{k}+r_{k+1} x_{0}^{p_{0}-1} \beta_{k}\right)^{2}\right)\right. \\
& +2 \sum_{l=k+1}^{n-1}\left(2^{2 p_{k} \cdots p_{l-1}-2} \bar{\varphi}_{k}^{2} \cdot \xi_{k}^{2 p_{k+1} \cdots p_{l-1}-2}\right. \\
& \left.+2^{2 p_{k+1} \cdots p_{l-1}-2} \beta_{k}^{2} \bar{\varphi}_{k+1}^{2} \xi_{k}^{2 p_{k+1} \cdots p_{l-1}-2}\right) \\
& \left.+2 k^{2}\left(\bar{\varphi}_{k}^{2}+\beta_{k}^{2} \bar{\varphi}_{k+1}^{2}\right)\right) \\
& +\xi_{n}^{2}\left(4-\frac{2}{p_{1} \cdots p_{n-1}}\right)^{2} \sum_{k=1}^{n-1} \sum_{l=1}^{k}\left(\frac{\partial \beta_{k}}{\partial z_{l}}\right)^{2} \dot{z}_{l}^{2} \\
& \leq \frac{1}{4} \sum_{k=1}^{n-1} \xi_{k}^{2}+\Lambda \xi_{n}^{2}\left(4-\frac{2}{p_{1} \cdots p_{n-1}}\right)^{2} \\
& \times\left(\sum_{k=1}^{n-1} 8\left(p_{1} \cdots p_{k-1} \beta_{k}\right)^{2}\right. \\
& \cdot\left(\left(2+2 \beta_{k}+2 r_{k} x_{0}^{p_{0}-1}\right)^{2}+\left(\beta_{k}+r_{k+1} x_{0}^{p_{0}-1} \beta_{k}\right)^{2}\right. \\
& +2 \sum_{l=k+1}^{n-1}\left(2^{2 p_{k} \cdots p_{l-1}-2} \bar{\varphi}_{k}^{2} \cdot \xi_{k}^{2 p_{k+1} \cdots p_{l-1}-2}\right. \\
& \left.+2^{2 p_{k+1} \cdots p_{l-1}-2} \beta_{k}^{2} \bar{\varphi}_{k+1}^{2} \xi_{k}^{2 p_{k+1} \cdots p_{l-1}-2}\right) \\
& \left.+2 k^{2}\left(\bar{\varphi}_{k}^{2}+\beta_{k}^{2} \bar{\varphi}_{k+1}^{2}\right)\right) \\
& \left.+\sum_{k=1}^{n-1} \sum_{l=1}^{k}\left(\frac{\partial \beta_{k}}{\partial z_{l}}\right)^{2} \dot{z}_{l}^{2}\right) \\
& \triangleq \frac{1}{4} \sum_{k=1}^{n-1} \xi_{k}^{2}+\Lambda \xi_{n}^{2} \varrho_{n 3}\left(x_{0}, z, \widehat{\Theta}\right) .
\end{aligned}
$$

In terms of (34), it can be seen that

$$
\begin{aligned}
& \frac{\partial W_{n}}{\partial \widehat{\Theta}} \dot{\bar{\Theta}} \\
& \quad \leq \gamma_{1}\left(4-\frac{2}{p_{1} \cdots p_{n-1}}\right)\left|\frac{\partial \alpha_{n-1}^{p_{1} \cdots p_{n-1}}}{\partial \widehat{\Theta}}\right|\left|\xi_{n}\right| \sum_{k=1}^{n-1} \xi_{k}^{2} \rho_{k}
\end{aligned}
$$

$$
\begin{aligned}
& \leq \frac{1}{4} \sum_{k=1}^{n-1} \xi_{k}^{2}+\xi_{n}^{2} \gamma_{1}^{2}\left(4-\frac{2}{p_{1} \cdots p_{n-1}}\right)^{2}\left|\frac{\partial \alpha_{n-1}^{p_{1} \cdots p_{n-1}}}{\partial \widehat{\Theta}}\right|^{2} \sum_{k=1}^{n-1} \xi_{k}^{2} \rho_{k}^{2} \\
& \triangleq \frac{1}{4} \sum_{k=1}^{n-1} \xi_{k}^{2}+\xi_{n}^{2} \varrho_{n 5}\left(x_{0}, z, \widehat{\Theta}\right) .
\end{aligned}
$$

Substituting (36)-(39) into (35), we have

$$
\begin{aligned}
\dot{V}_{n} \leq & -x_{0}^{p_{0}+1}-\frac{1}{\theta_{1}} \sum_{k=1}^{n-1} \xi_{k}^{2}+\Lambda \xi_{n}^{2}\left(\varrho_{n 1}+\varrho_{n 2}+\varrho_{n 3}+\varrho_{n 4}\right) \\
& +\xi_{n}^{2} \varrho_{n 5}+\left|\xi_{n}\right|^{2-\left(1 / p_{1} \cdots p_{n-1}\right)}\|\psi\| \\
& +\beta(t) \xi_{n}^{2-\left(1 / p_{1} \cdots p_{n-1}\right)} u^{p_{n}}+\frac{1}{\gamma_{2}} \widetilde{\Lambda} \dot{\bar{\Lambda}}
\end{aligned}
$$

with $\varrho_{n 4}\left(x_{0}, z, \widehat{\Theta}\right)=0$. Denote

$$
\begin{gathered}
\rho_{n}\left(x_{0}, z, \widehat{\Theta}\right)=\varrho_{n 1}+\varrho_{n 2}+\varrho_{n 3}+\varrho_{n 4}, \\
\omega=\gamma_{2} \xi_{n}^{2} \rho_{n}\left(x_{0}, z, \widehat{\Theta}\right) .
\end{gathered}
$$

As a result, (40) can be further transformed into

$$
\begin{aligned}
\dot{V}_{n} \leq & -x_{0}^{p_{0}+1}-\frac{1}{\theta_{1}} \sum_{k=1}^{n-1} \xi_{k}^{2}+\beta(t) \xi_{n}^{2-\left(1 / p_{1} \cdots p_{n-1}\right)} u^{p_{n}} \\
& +\xi_{n}^{2}\left(\rho_{n} \widehat{\Lambda}+\varrho_{n 5}\right)+\left|\xi_{n}\right|^{2-\left(1 / p_{1} \cdots p_{n-1}\right)}\|\psi\| \\
& +\frac{1}{\gamma_{2}} \widetilde{\Lambda}(\omega-\dot{\bar{\Lambda}}) .
\end{aligned}
$$

Inspired by $[6,16]$, the error variable $\xi_{n}=z_{n}^{p_{1} \cdots p_{n-1}}-$ $\alpha_{n-1}^{p_{1} \cdots p_{n-1}}$ can be viewed as an adaptive sliding surface in the $x$-coordinate. Denote the sliding manifold by $S=\xi_{n}$, then the adaptive sliding mode control and the parameter update law can be chosen as

$$
\begin{aligned}
u^{p_{n}}=-\frac{1}{\beta}\left(\xi_{n}^{1 / p_{1} \cdots p_{n-1}}\right. & \\
& \quad \times\left(\rho_{n}\left(x_{0}, z, \widehat{\Theta}\right) \sqrt{1+\widehat{\Lambda}^{2}}+\rho_{n 5}\left(x_{0}, z, \widehat{\Theta}\right)\right) \\
& -\operatorname{sgn}(S)(k+\|\psi\|)), \\
\dot{\Lambda}=\omega, &
\end{aligned}
$$

where $k>0$ is a constant and $\operatorname{sgn}(\cdot)$ is the sign function. Accordingly, we obtain

$$
\dot{V}_{n} \leq-x_{0}^{p_{0}+1}-\frac{1}{\theta_{1}} \sum_{k=1}^{n-1} \xi_{k}^{2}-k|S|^{2-\left(1 / p_{1} \cdots p_{n-1}\right)} .
$$

Since $V_{n}$ is a positive definite and proper Lyapunov function, (45) guarantees the convergence of the system 
trajectory to the origin of the sliding surface $S=\xi_{n}$ in finite time. In addition, the convergence properties of the states and the parameter estimate remain unchanged with respect to those of the standard backstepping design procedure. That is, the states $\left(x_{0}(t), x(t)\right)$ of system (1) converge to zero asymptotically and the parameter estimates $\widehat{\Theta}(t), \widehat{\Lambda}(t)$ are bounded.

\section{Switching Control Strategy}

In this section, we consider the case of $x_{0}\left(t_{0}\right)=0$. Without loss of generality, we assume that $t_{0}=0$. As to the case of $x_{0}(0) \neq 0$, we have given the controller (5) and (43) for $u_{0}$ and $u$ of system (1), respectively. Now, we turn to how to select control laws while $x_{0}(0)=0$.

We consider the constant control if $\left|x_{0}\right|$ is small enough. When $x_{0}(0)=0$, we choose $u_{0}$ as follows:

$$
u_{0}(0)=u_{0}^{*}, \quad u_{0}^{*}>0 \text {. }
$$

In accordance with the first subsystem of (1), we know that

$$
\dot{x}_{0}(0)=d_{0}(0) u_{0}^{p_{0}}(0)=d_{0}(0) u_{0}^{* p_{0}}>0 \text {. }
$$

So, for any small enough constant $c_{0}^{*}$, there exists an instant $t_{s}^{*}>0$ such that $x_{0}\left(t_{s}^{*}\right)=c_{0}^{*}$. During the finite time interval $\left[0, t_{s}^{*}\right)$, a new adaptive sliding mode control $u=u^{*}\left(x_{0}, x\right)$ using $u_{0}$ as defined in (46) and two new parameter update laws can be obtained following the procedure described in the previous section. Combining with $x_{0}\left(t_{s}^{*}\right)=c_{0}^{*} \neq 0$, we can switch the control inputs $u_{0}$ and $u$ into (5) and (43), respectively.

Now we state the main result of this paper.

Theorem 6. Suppose that Assumptions 1-3 are satisfied. If the proposed control design procedure together with the previous switching control strategy is applied to system (1), then, for any initial conditions in the state space $\Omega=\left\{\left(x_{0}, x\right) \in \mathbb{R}^{n+1}\right\}$, system (1) will be asymptotically stabilized at the equilibrium, and specifically, the states are regulated to the sliding surface $S=0$ in finite time while keeping the estimated parameters bounded.

\section{Simulation Example}

Consider the following example:

$$
\begin{gathered}
\dot{x}_{0}=d_{0}(t) u_{0}^{3}, \\
\dot{x}_{1}=d_{1}(t) x_{2}^{3} u_{0}^{2}+\frac{1}{4} x_{1}, \\
\dot{x}_{0}=u^{3},
\end{gathered}
$$

where $x=\left(x_{0}, x_{1}, x_{2}\right)^{T}$ is the state, $u_{0}$ and $u$ are the control inputs of the system, $0<\theta_{1} \leq d_{1}(t), d_{2}(t) \leq \theta_{2}$ are unknown control coefficients with $\theta_{1}$ and $\theta_{2}$ being unknown positive constants. It can be seen that system (48) is in the form of (1) with $p_{0}=p_{1}=p_{2}=3, q_{1}=2, \phi_{2}\left(x_{0}, x_{1}, x_{2}\right)=(1 / 4) x_{2}$.

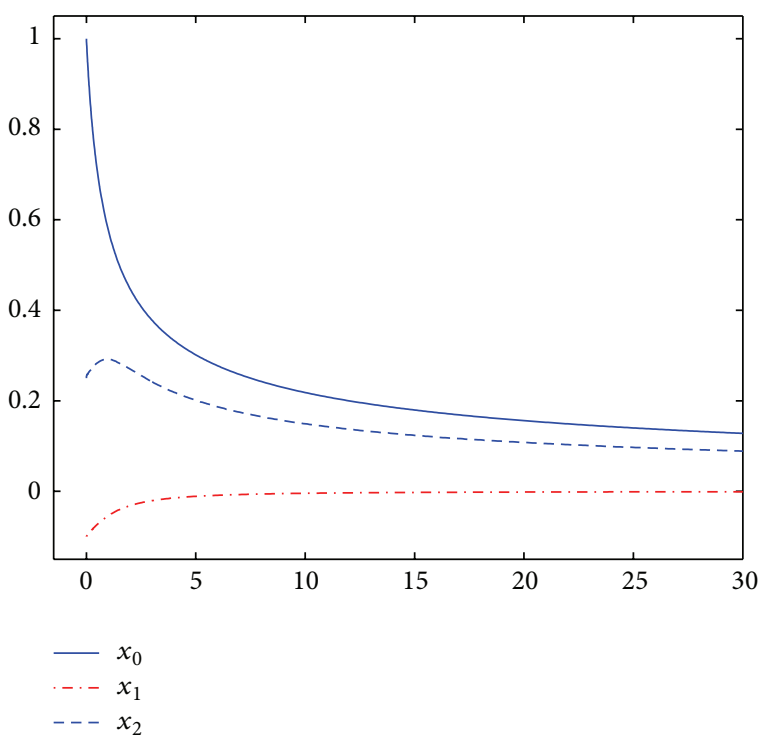

Figure 1: States of the closed-loop system.

Denote $\widehat{\Theta}$ as the estimation of $\Theta=\left(1+\theta_{2}^{6}\right) \max \left\{1, \theta_{1}^{-1}\right\}$ and $\Lambda=\max \left\{\theta_{2}, \theta_{2}^{6}\right\}$.

Here, we just consider the case of $x_{0}(0) \neq 0$. In the first subsystem of (48), we choose the control law

$$
u_{0}=-x_{0}
$$

Using the developed design procedure in Section 3, we can obtain that

$$
\begin{aligned}
& u^{3}=-\xi_{2}^{1 / 3}\left(\rho_{2}\left(x_{0}, x_{1}, x_{2}, \widehat{\Theta}\right) \sqrt{1+\widehat{\Lambda}^{2}}\right. \\
& \left.\quad+\varrho_{25}\left(x_{0}, x_{1}, x_{2}, \widehat{\Theta}\right)\right) \\
& \quad-k \operatorname{sgn}(S), \\
& \dot{\widehat{\Theta}}=\gamma_{1} \xi_{1}^{2} \rho_{1}, \\
& \dot{\widehat{\Lambda}}=\gamma_{2} \xi_{2}^{2} \rho_{2},
\end{aligned}
$$

where $\xi_{1}=x_{1} / u_{0}^{2}, \rho_{1}=9 / 4+2 x_{0}^{2}, \beta_{1}=\rho_{1}\left(1+\widehat{\Theta}^{2}\right)^{1 / 2}, \xi_{2}=x_{2}^{3}+$ $\xi_{1} \beta_{1}, S=\xi_{2}, \rho_{2}=1+(40 / 3)^{4} x_{0}^{4} \xi_{1}^{4} \xi_{2}^{2}\left(1+\widehat{\Theta}^{2}\right)^{2}+100 / 3 \beta_{1}^{2}\left(\beta_{1}^{2}+\right.$ $\left.4 x_{0}^{4}+1 / 16\right)+10 / 3 \beta_{1}, \varrho_{25}=(100 / 9) \gamma_{1}^{2} \xi_{1}^{4} \rho_{1}^{4}, k$, and $\gamma_{1}$ and $\gamma_{2}$ are positive design parameters.

Let the unknown functions be $d_{0}(t)=1-(1 / 2) \cos t$, $d_{1}(t)=1+(1 / 4) \sin t$. The unknown constants can be chosen as $\theta_{1}=1 / 2$ and $\theta_{2}=3 / 2$. Take $k=\gamma_{1}=\gamma_{2}=1$. Simulation results of the closed-loop system are shown in Figure 1 with the initial condition $\left(x_{0}(0), x_{1}(0), x_{2}(0)\right)=(1,-0.1,0.25)$.

\section{Conclusions}

In this paper, the global stabilization problem is considered for a class of uncertain high-order nonholonomic systems with unknown control coefficients. By adding a power integrator technique, we propose a combined adaptive/sliding 
mode control scheme. To get around the stabilization burden associated with the nonholonomic control systems, a switching control strategy is exploited in this procedure. The designed adaptive sliding mode controller guarantees the global asymptotical stabilization of the closed-loop system as well as the boundedness of the parameter estimates. Moreover, the attractiveness of the sliding surface $S=0$ in finite time is also obtained with the help of the proposed control approach.

\section{Acknowledgments}

This work is supported by the National Natural Science Foundation of China (nos. 61273091, 61203013, and 61004003), the Shandong Provincial Natural Science Foundation of China (no. ZR2011FM033), the Outstanding Middle-Age and Young Scientist Award Foundation of Shandong Province (no. BS2011DX012), and the Fundamental Research Funds for the Central Universities of China (no. CXLX12_0097).

\section{References}

[1] I. Kolmanovsky and N. H. McClamroch, "Developments in nonholonomic control problems," IEEE Control Systems Magazine, vol. 15, no. 6, pp. 20-36, 1995.

[2] R. W. Brockett, "Asymptotic stability and feedback stabilization," in Differential Geometric Control Theory, R. W. Brockett, R. S. Millman, and H. J. Sussmann, Eds., vol. 27 of Progress in Mathematics, pp. 181-191, Birkhäuser, Boston, Mass, USA, 1983.

[3] Y. Wu, B. Wang, and G. D. Zong, "Finite-time tracking controller design for nonholonomic systems with extended chained form," IEEE Transactions on Circuits and Systems II, vol. 52, no. 11, pp. 798-802, 2005.

[4] T. Floquet, J.-P. Barbot, and W. Perruquetti, "Higher-order sliding mode stabilization for a class of nonholonomic perturbed systems," Automatica, vol. 39, no. 6, pp. 1077-1083, 2003.

[5] G. Bartolini, A. Ferrara, and E. Usai, "Output tracking control of uncertain nonlinear second-order systems," Automatica, vol. 33, no. 12, pp. 2203-2212, 1997.

[6] G. Bartolini, A. Ferrara, L. Giacomini, and E. Usai, "Properties of a combined adaptive/second-order sliding mode control algorithm for some classes of uncertain nonlinear systems," IEEE Transactions on Automatic Control, vol. 45, no. 7, pp. 13341341, 2000.

[7] A. Ferrara, L. Giacomini, and C. Vecchio, "Control of nonholonomic systems with uncertainties via second-order sliding modes," International Journal of Robust and Nonlinear Control, vol. 18, no. 4-5, pp. 515-528, 2008.

[8] A. Ferrara, L. Giacomini, and C. Vecchio, "Stabilization of nonholonomic uncertain systems via adaptive second order sliding mode control," in Modern sliding mode control theory, vol. 375 of Lecture Notes in Control and Information Sciences, pp. 223-245, Springer, Berlin, Germany, 2008.

[9] L. Yang and J. Yang, "Stabilization for a class of nonholonomic perturbed systems via robust adaptive sliding mode control," in Proceedings of the American Control Conference (ACC '10), pp. 1178-1183, Baltimore, Md, USA, July 2010.

[10] W. Lin and R. Pongvuthithum, "Discontinuous controllers for high-order nonholonomic systems in power chained form," in Proceedings of the American Control Conference (ACC '10), pp. 3254-3258, Chicago, Ill, USA, June 2000.
[11] W. Lin and C. Qian, "Adding one power integrator: a tool for global stabilization of high-order lower-triangular systems," Systems \& Control Letters, vol. 39, no. 5, pp. 339-351, 2000.

[12] W. Lin, R. Pongvuthithum, and C. Qian, "Control of high-order nonholonomic systems in power chained form using discontinuous feedback," IEEE Transactions on Automatic Control, vol. 47, no. 1, pp. 108-115, 2002.

[13] X.-Y. Zheng and Y.-Q. Wu, "Controller design of high order nonholonomic system with nonlinear drifts," International Journal of Automation and Computing, vol. 6, no. 3, pp. 240-244, 2009.

[14] Z. Zhang, S. Xu, and B. Wang, "Adaptive actuator failure compensation with unknown control gain signs," IET Control Theory \& Applications, vol. 5, no. 16, pp. 1859-1867, 2011.

[15] J. Zhang and Y. Liu, "Adaptive stabilization of high-order nonholonomic systems with unknown control coefficients," in Proceedings of the 30th Chinese Control Conference (CCC '11), pp. 538-543, Yantai, China,, July 2011.

[16] H. Sira-Ramirez, M. Zribi, and S. Ahmad, "Adaptive dynamical feedback regulation strategies for linearizable uncertain systems," International Journal of Control, vol. 57, no. 1, pp. 121-139, 1993.

[17] Z. Q. Zhang, J. W. Lu, and S. Y. Xu, "Tuning functions-based robust adaptive tracking control of a class of nonlinear systems with time delays," International Journal of Robust and Nonlinear Control, vol. 22, no. 14, pp. 1631-1646, 2012. 


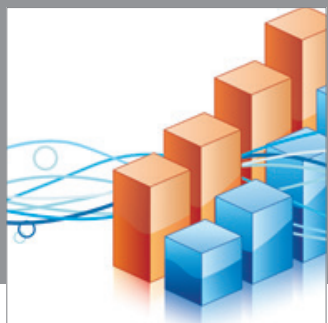

Advances in

Operations Research

mansans

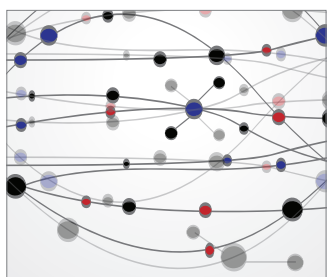

The Scientific World Journal
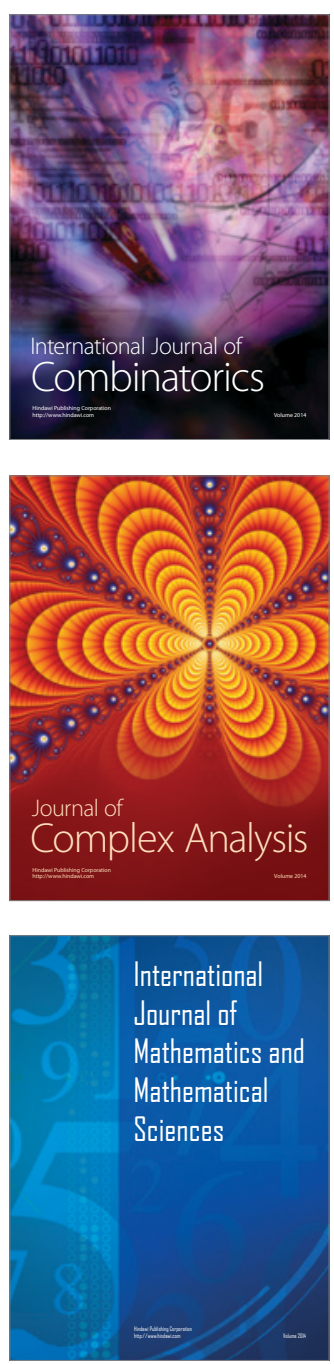
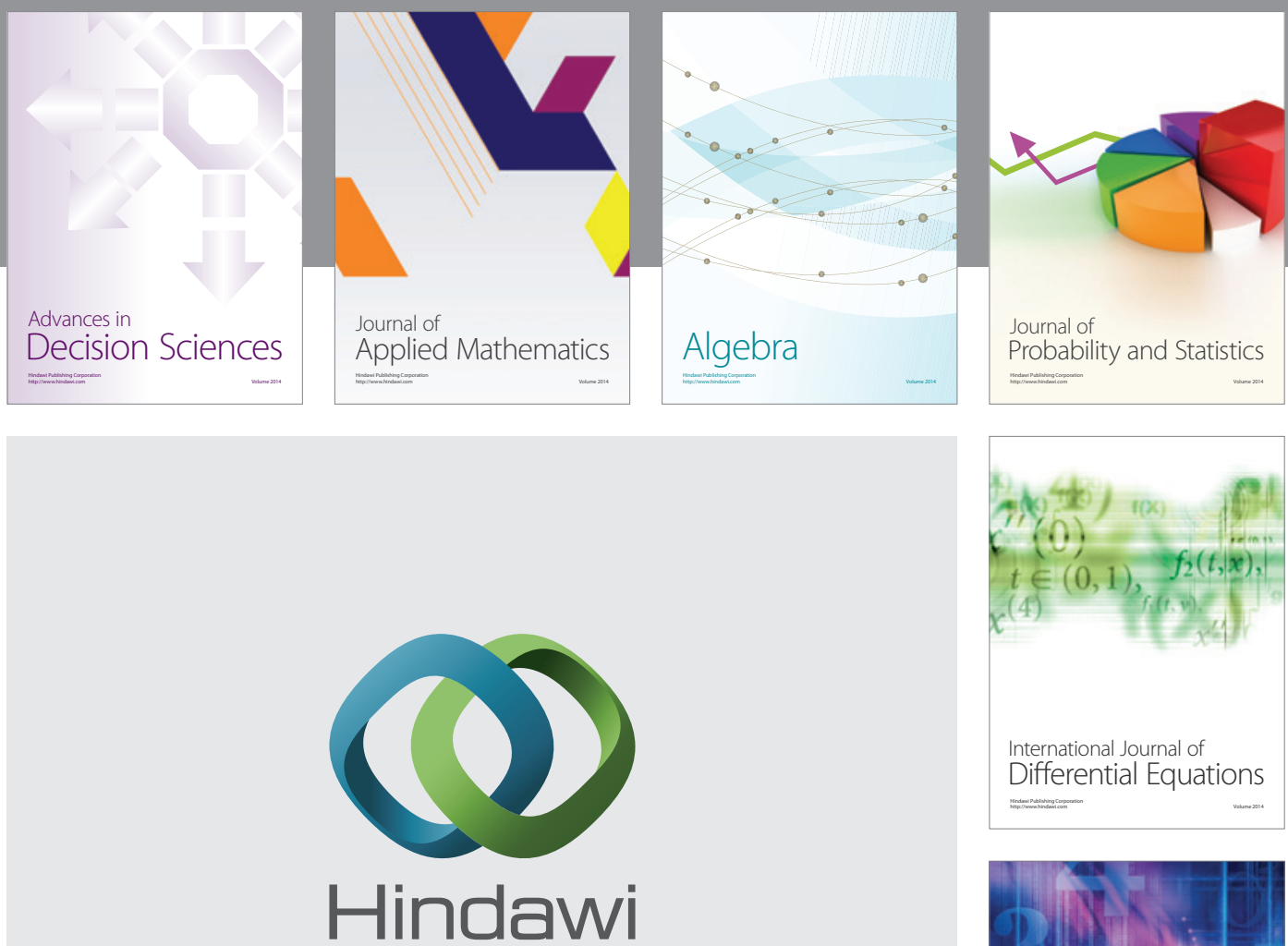

Submit your manuscripts at http://www.hindawi.com
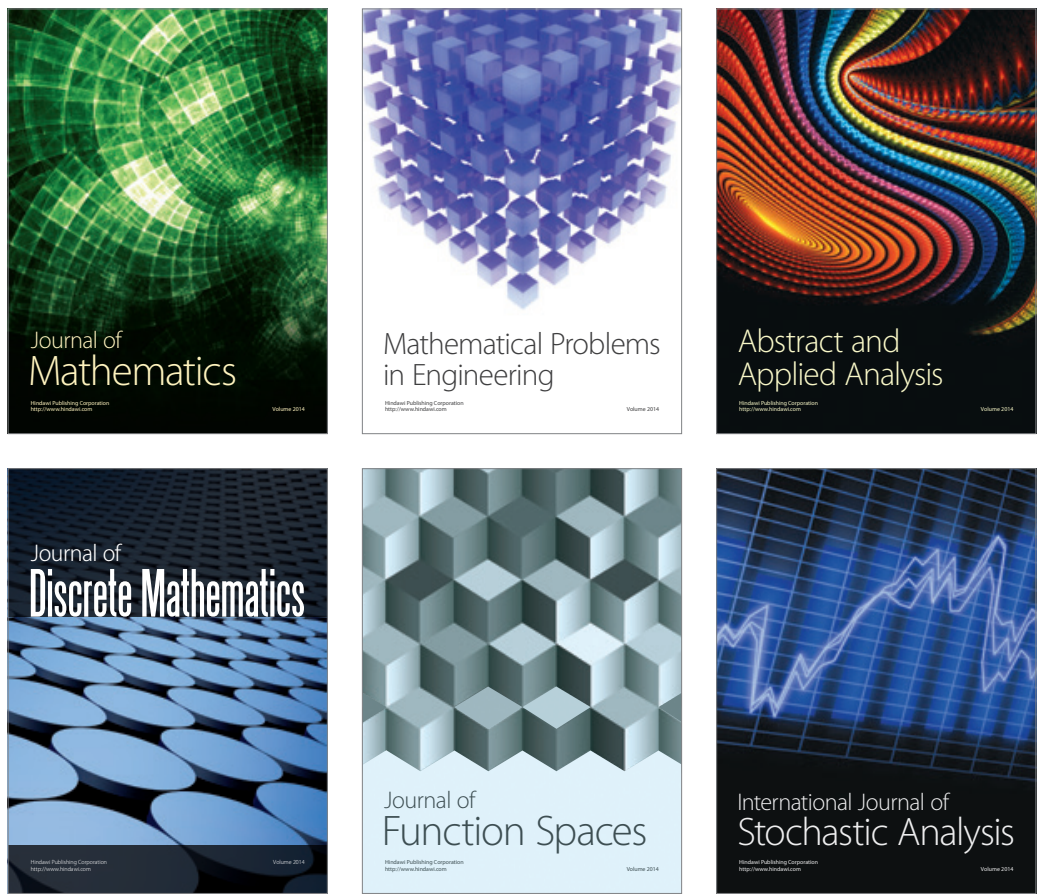

Journal of

Function Spaces

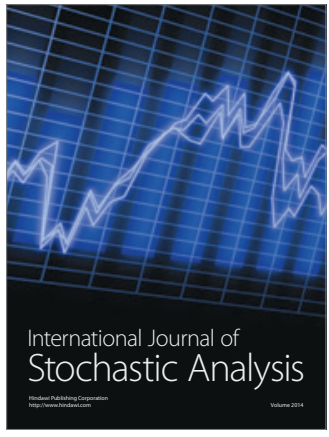

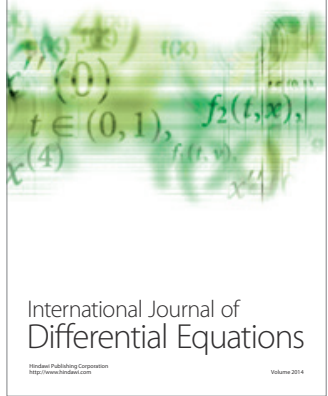
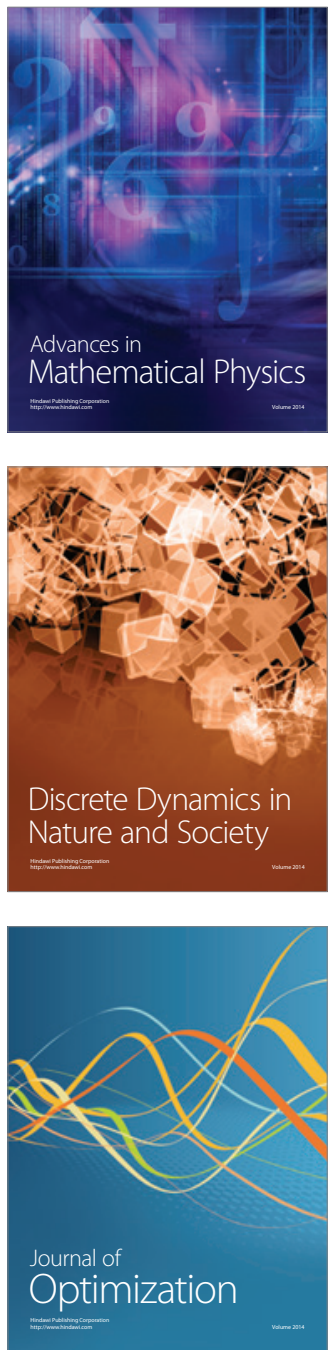